\title{
Review Article \\ Diagnosis Value of the Serum Amyloid A Test in Neonatal Sepsis: A Meta-Analysis
}

\author{
Haining Yuan, ${ }^{1}$ Jie Huang, ${ }^{2}$ Bokun Lv, ${ }^{1}$ Wenying Yan, ${ }^{1,3}$ Guang Hu, \\ Jian Wang, ${ }^{2}$ and Bairong Shen ${ }^{1,3}$ \\ ${ }^{1}$ Center for Systems Biology, Soochow University, Suzhou 215006, China \\ ${ }^{2}$ Affiliated Children's Hospital of Soochow University, Suzhou 225121, China \\ ${ }^{3}$ Suzhou Zhengxing Translational Biomedical Informatics Ltd., Taicang 215400, China \\ Correspondence should be addressed to Guang Hu; huguang@suda.edu.cn
}

Received 29 April 2013; Accepted 4 July 2013

Academic Editor: Zhongming Zhao

Copyright (C) 2013 Haining Yuan et al. This is an open access article distributed under the Creative Commons Attribution License, which permits unrestricted use, distribution, and reproduction in any medium, provided the original work is properly cited.

\begin{abstract}
Neonatal sepsis (NS), a common disorder for humans, is recognized as a leading global public health challenge. This meta-analysis was performed to assess the accuracy of the serum amyloid A (SAA) test for diagnosing NS. The studies that evaluated the SAA test as a diagnotic marker were searched in Pubmed, EMBASE, the Cochrane Library, and Google Network between January 1996 and June 2013. A total of nine studies including 823 neonates were included in our meta-analysis. Quality of each study was evaluated by the quality assessment of diagnostic accuracy studies tool (QUADAS). The SAA test showed moderate accuracy in the diagnosis of NS both at the first suspicion of sepsis and 8-96 h after the sepsis onset, both with $Q^{*}=0.91$, which is similar to the PCT and CRP tests for the diagnosis of NS in the same period. Heterogeneity between studies was also explained by cut-off point, SAA assay, and age of included neonates. On the basis of our meta-analysis, therefore, SAA could be promising and meaningful in the diagnosis of NS.
\end{abstract}

\section{Introduction}

Neonatal sepsis (NS) is recognized as a leading global public health challenge because of the important contribution to neonatal morbidity and mortality in both low and high income countries [1-3]. It is mainly responsible for most of neonatal deaths, and the incidence of NS is about 3-40 per 1000 live births, the mortality rate of whom varies from $9 \%$ to $20 \%$ of affected neonates $[4,5]$.

Neonatal sepsis may be defined, both clinically and/or microbiologically, by positive blood and/or cerebrospinal fluid cultures. Early gradual signs and symptoms of NS are often indefinite and subtle, especially at the onset, which is easily confused with other common noninfectious causes [6]. Clinicians often treat with a broad-spectrum antibiotic and prolong treatment with empirical antibiotics, which are associated with adverse outcomes. Furthermore, physical signs are used to identify neonates at risk of sepsis with limited specificity, generally resulting in large number of unnecessary referrals, and antibiotics treatment in the setting of negative cultures may not be benign $[7,8]$. Early diagnosis and treatment are vital to improve gradual outcomes; if diagnosed early and with aggressive supportive care, it is possible to save most cases of NS [9]. To this aim, clinicians have sought reliable markers to detect early NS for a long time and to exclude diseases of noninfectious origin $[10,11]$. Till now, a large number of markers have been proposed for early diagnosis of sepsis [12], especially C-reactive protein (CRP) and procalcitonin (PCT). CRP is an acute-phase protein found in the blood that is produced by the liver because of infection or tissue injury, while PCT is a 116-amino acid peptide involved as a precursor in calcium homeostasis, and both of them have been widely used as useful markers for the diagnosis of neonatal sepsis [13-17]. However, the specificity and the value of CRP and PCT still have challenges; thus, there is a continuous need for searching for better biomarkers of sepsis.

Serum amyloid A (SAA), the precursor protein in inflammation-associated reactive amyloidosis, whose level in the 
blood increases up to 1000 fold in response to information, is synthesized in the liver. SAA is also an acute phase reactant like PCT and CRP, which has been proven to be a prognostic marker in late-onset sepsis in preterm infants [18-20]. Arnon et al. [21] reported that SAA had an overall better diagnostic accuracy than CRP for predicting early onset sepsis. Also, they showed that SAA was a useful inflammatory marker during late-onset sepsis in preterm infants [22]. However, some studies showed an opposite opinion $[23,24]$. In view of this contradiction, a more comprehensive study is needed to discuss the accuracy of the SAA test for the diagnosis of NS.

Our primary objective is to systematically and quantitatively evaluate all published studies about the diagnostic use of SAA for NS.

\section{Methods}

2.1. Retrieval and Selection of Studies. The common approach of computer-aided literature search is used to search PUBMED, EMBASE (http://www.embase.com/), the Cochrane Library (http://www.thecochranelibrary.com/ view/0/index.html), and Google Network for relevant citations from January 1996 to June 2013. Our search terms included "serum amyloid A," "SAA," "sepsis," "septicemia," "neonate," "newborn," "infant," and mutual combinations. We have examined the references of known articles to fully retrieve.

The following criteria were applied to identify studies for inclusion in our meta-analysis: (1) studies that assessed the diagnostic accuracy of the SAA test on NS; (2) studies providing both sensitivity and specificity or sufficient information to construct the $2 \times 2$ contingency tables; and (3) studies with the sample containing only neonates. Articles including studies that evaluated SAA levels as diagnostic markers for NS are appropriate. The gold standard for the diagnosis of neonatal sepsis is microbial culture blood or other sterile body fluids in these included studies. Furthermore, the change of the SAA in the research sample is the index test for the diagnosis of neonatal sepsis. Selection of articles was performed by two investigators independently to ensure the high accuracy.

2.2. Data Extraction. We extracted data from selected articles, which include first authors, years of publication, study population, region, methods of SAA assay, diagnostic cutoff point and time, and methods quality. Accurate data was extracted to construct $2 \times 2$ table at a specific time.

2.3. Quality Assessment. Quality assessment of studies was performed based on the quality assessment of diagnostic accuracy studies (QUADAS) tool [25]. This tool consists of 11 key items. Each item was assessed by scoring it as "low," "high," "unclear," which are phrased to the answer, such as "yes" indicates low risk of bias.

2.4. Statistical Analysis. The statistical analysis was performed using Review Manager 5.0 and Meta-DiSc 1.4 software. Studies included in the meta-analysis were divided into two groups according to the time of SAA test for diagnosis of NS. The neonates at the onset of sepsis were set as a group, and those 8-96 h after onset were set as another. The sensitivity, specificity, and diagnostic odds ratio (OR) with corresponding $95 \%$ confidence intervals (CI) were calculated for each study. Meanwhile, the pooled sensitivity, specificity and diagnosis OR were also calculated for each group. The diagnostic OR expresses how much greater are the odds of having sepsis for neonates with a positive test result than for neonates with a negative test result [26]. Heterogeneity among included studies is assessed by using the Cochrane $Q$ statistic and quantified with the $I^{2}$ lying between $0 \%$ and $100 \%$ [27]. In general, $I^{2}(>50 \%)$ shows that heterogeneity among studies produce some impact, whereas $I^{2} \quad(<50 \%)$ shows that homogeneity is good for the reliability of metaanalysis. We further performed sensitivity analysis to explore the reasons of heterogeneity and examined characteristics of included studies. To summarize these results, we constructed a summary receiver operator characteristic (SROC) curve, which shows the relationship between sensitivity and the proportion of false positives (1-specificity). $Q^{*}$ values, defined by the point where sensitivity equals specificity, were calculated from the SROC curves. Meanwhile, the area under SROC curve (AUC) was also calculated to show the probability of the correctly ranked diagnostic test values for a random pair of diseased and nondiseased subjects [28].

\section{Results}

3.1. Characteristics and Quality of the Included Studies. The literature search was performed as described, and 57 potentially relevant articles were identified. Only 9 articles met our inclusion criteria. Figure 1 shows the process of selecting studies. Detailed characteristics and data of each included study are presented in Tables 1 and 2, respectively.

All the conditions and methods of the included studies, as shown in Figure 2, were used for different quality assessment of diagnostic accuracy. Each included study was strictly judged on the basis of 11 QUADAS tool criteria [25]: withdrawals explained; uninterpretable results reported; relevant clinical information; index test results blinded; reference standard results blinded; incorporation avoided; differential verification avoided; partial verification avoided; Acceptable delay between tests; acceptable reference standard; and representative spectrum. We also appraise them quantitatively according to 11 QUADAS tool criteria, as shown in Table 2.

3.2. Accuracy of the SAA Test in the Diagnosis of NS. Nine articles, which meet inclusion criteria, estimate the use of the SAA test in the diagnosis of NS. We set studies at the first suspicion of NS as a group and those at 8-96 h after sepsis onset as another group on the basis of time point for the SAA test.

Nine studies from included papers evaluated the use of the SAA test at the first suspicion of sepsis. The sensitivity ranged from $23 \%$ to $100 \%$ (pooled sensitivity: $0.84,95 \% \mathrm{CI}$ $80 \%-87 \%$ ), whereas specificity ranged from $44 \%$ to $100 \%$ (pooled sensitivity: 0.89 , 95\% CI 86\%-92\%). The detailed descriptions are shown in Figure 3. We found significant heterogeneity among studies (sensitivity, $I^{2}=92.7 \%$; specificity, 
TABLE 1: Characteristics of studies included in the meta-analysis of the diagnosis of neonatal sepsis using a SAA test.

\begin{tabular}{|c|c|c|c|c|c|}
\hline Study and year & Study population & Patients $(n)$ & Region & Assay method & $\begin{array}{l}\text { Time of SAA test: } \\
\text { cutoff }(\mathrm{mg} / \mathrm{L})\end{array}$ \\
\hline \multirow[t]{2}{*}{ Enguix et al. 2001 [31] } & $\begin{array}{l}\text { Cases: NICU neonates with } \\
\text { sepsis }\end{array}$ & 46 & Spain & AMLN & Onset: 41.3 \\
\hline & $\begin{array}{l}\text { Control: neonates without } \\
\text { sepsis }\end{array}$ & & & & \\
\hline Arnon et al. 2007 [21] & $\begin{array}{l}\text { Cases: full-term neonates } \\
\text { with sepsis } \\
\text { Control: neonates without } \\
\text { sepsis }\end{array}$ & 104 & Israel & ALPIA & $\begin{array}{c}\text { Onset: } 8 \\
24 \text { h after the onset: } 10\end{array}$ \\
\hline Çetinkaya et al. 2009 [29] & $\begin{array}{l}\text { Cases: NICU neonates with } \\
\text { probable sepsis } \\
\text { Control: neonates without } \\
\text { sepsis }\end{array}$ & 163 & Turkey & INMM & $\begin{array}{c}\text { Onset: } 68 \\
48 \text { h after the onset: } 68\end{array}$ \\
\hline Yildiz et al. 2008 [23] & $\begin{array}{l}\text { Cases: NICU newborns } \\
\text { with suspected sepsis } \\
\text { control: noninfected } \\
\text { newborns }\end{array}$ & 72 & Turkey & ELISA & $\begin{array}{c}\text { Onset: } 5.5 \\
96 \text { h after the onset: } 5.7\end{array}$ \\
\hline Arnon et al. 2005 [22] & $\begin{array}{l}\text { Cases: neonates with } \\
\text { proven or clinical sepsis } \\
\text { Control: noninfected } \\
\text { newborns }\end{array}$ & 116 & Israel & ELISA & $\begin{array}{l}\text { Onset: } 10 \\
8 \mathrm{~h} \text { after the onset: } 10\end{array}$ \\
\hline Arnon et al. 2002 [44] & $\begin{array}{l}\text { Cases: preterm infants with } \\
\text { sepsis or suspected sepsis } \\
\text { Control: healthy preterm } \\
\text { infants }\end{array}$ & 94 & Israel & ELISA & Onset: 10 \\
\hline Edgar et al. 2010 [24] & $\begin{array}{l}\text { Cases: term/preterm } \\
\text { neonates with infection } \\
\text { Control: term/preterm } \\
\text { neonates with infection }\end{array}$ & 68 & England & ELISA & Onset: 1 \\
\hline Mostafa et al. 2011 [45] & $\begin{array}{l}\text { Cases: infants with sepsis or } \\
\text { suspected sepsis } \\
\text { Control: healthy neonates }\end{array}$ & 100 & Egypt & ELISA & Onset: $>10$ \\
\hline Mohsen et al. 2012 [30] & $\begin{array}{l}\text { Cases: term/preterm } \\
\text { neonate with sepsis } \\
\text { Control: healthy neonates }\end{array}$ & 60 & Egypt & ELISA & $\begin{array}{l}\text { Onset: cases, } 40.16 \pm 35.17 \\
\text { control, } 6.45 \pm 2.42\end{array}$ \\
\hline
\end{tabular}

AMLN: automatic laser nephelometry; ALPIA: automated latex photometric immunoassay; INMM: immunonephelometric method; ELISA: enzyme-linked immunoassay; HSAIA: highly sensitive automated immunoassays, and NICU: neonatal intensive care unit.

TABle 2: True positive, Fp, Fn, Tn, Se, Sp, time, and QUADAS of included studies for the diagnosis of NS.

\begin{tabular}{|c|c|c|c|c|c|c|c|c|}
\hline Study and year & $\mathrm{Tp}$ & $\mathrm{Fp}$ & Fn & $\mathrm{Tn}$ & $\mathrm{Se}$ & Sp & Time & QUADAS \\
\hline Enguix et al. 2001 [31] & 19 & 2 & 1 & 24 & 0.95 & 0.92 & Onset & 9 \\
\hline \multirow[t]{2}{*}{ Arnon et al. 2007 [21] } & 22 & 4 & 1 & 77 & 0.96 & 0.95 & Onset & 7 \\
\hline & 22 & 2 & 1 & 79 & 0.96 & 0.98 & $24 \mathrm{~h}$ after the onset & \\
\hline \multirow[t]{2}{*}{ Çetinkaya et al. 2009 [29] } & 94 & 0 & 29 & 40 & 0.76 & 1 & Onset & 6 \\
\hline & 80 & 0 & 41 & 42 & 0.66 & 1 & $48 \mathrm{~h}$ after the onset & \\
\hline \multirow[t]{2}{*}{ Yildiz et al. 2008 [23] } & 27 & 20 & 9 & 16 & 0.75 & 0.44 & Onset & 5 \\
\hline & 31 & 24 & 5 & 12 & 0.86 & 0.33 & $96 \mathrm{~h}$ after the onset & \\
\hline \multirow[t]{2}{*}{ Arnon et al. 2005 [22] } & 40 & 5 & 2 & 68 & 0.95 & 0.93 & Onset & 8 \\
\hline & 37 & 12 & 0 & 67 & 1 & 0.85 & $8 \mathrm{~h}$ after the onset & \\
\hline Arnon et al. 2002 [44] & 42 & 4 & 0 & 48 & 1 & 0.92 & Onset & 6 \\
\hline Edgar et al. 2010 [24] & 6 & 3 & 20 & 39 & 0.23 & 0.93 & Onset & 8 \\
\hline Mostafa et al. 2011 [45] & 80 & 2 & 2 & 16 & 0.98 & 0.89 & Onset & 6 \\
\hline Mohsen et al. 2012 [30] & 26 & 2 & 4 & 28 & 0.87 & 0.93 & Onset & 6 \\
\hline
\end{tabular}

Tp: true positive; Fp: false positive; Fn: false negative; Tn: true negative; Se: sensitivity; and Sp: specificity. 


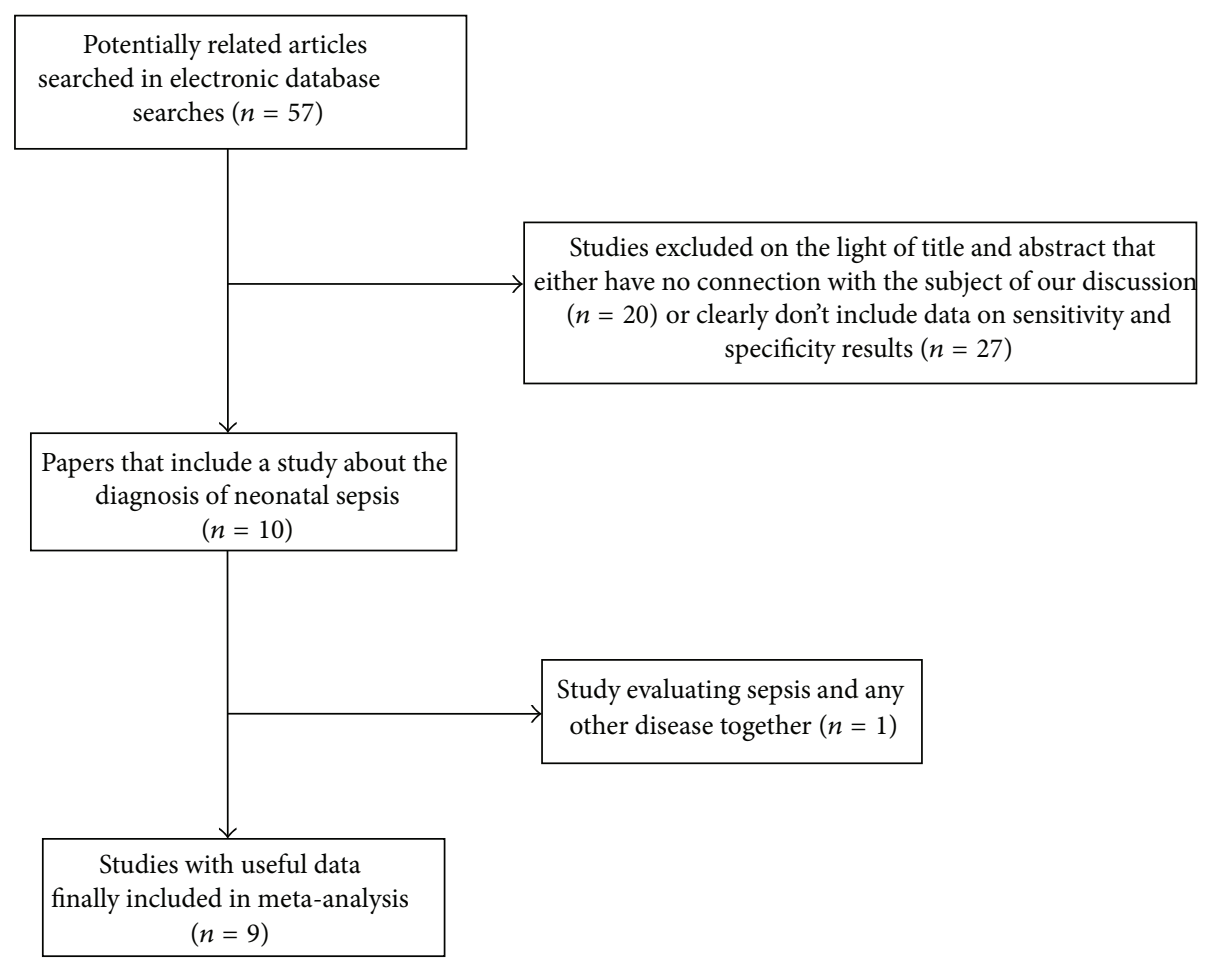

FIGURE 1: Flow chart of study evaluation and inclusion in the meta-analysis of studies involving diagnosis of neonatal sepsis using a SAA test.

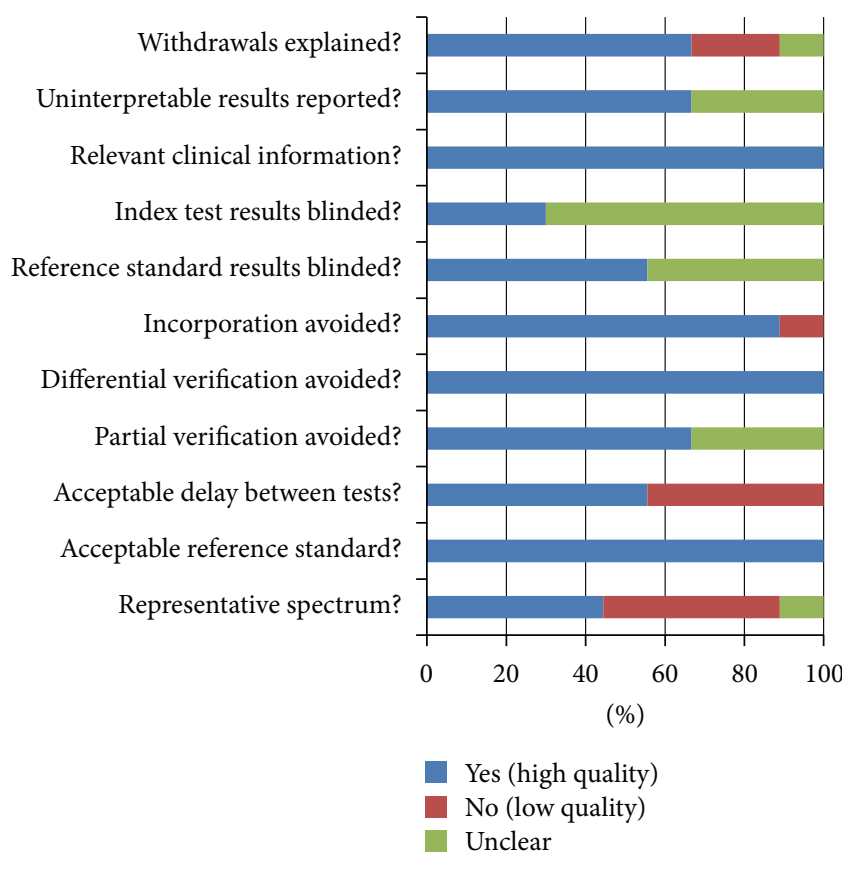

FIGURE 2: Summary of the methodological quality assessment of the included studies according to 11 QUADAS tool criteria, which are presented as percentages.

$\left.I^{2}=86.5 \%\right)$, which indicated that patient selection or other covariates might be responsible for heterogeneity.

The value of DOR of SAA was 91.84 (95\% CI, 16.78502.80 ), as shown in the forest plot of Figure 4. Among these studies, we also detected significant heterogeneity $\left(I^{2}=86.8 \%\right)$. The corresponding SROC curve was plotted in Figure 5, which shows the AUC was 0.96 with standard error of 0.02 , and the pooled diagnostic accuracy $\left(Q^{*}\right)$ was 0.90 with standard error of 0.03 , showing a high overall accuracy of SAA for NS.

Four studies from included papers evaluated the use of the SAA test at 8-96h after the first suspicion of sepsis. Accordingly, Figure 6 shows the pooled sensitivity and specificity, and the diagnostic OR and SROC curve were shown in Figures 7 and 5, respectively.

3.3. Comparison of the Diagnostic Accuracy of Markers for NS. CRP and PCT have been proved to be useful biomarkers for the diagnosis of neonatal sepsis [15-17]. To show the value of diagnosis of the SAA test for NS, we compared SAA with CRP and PCT. Six trials evaluated the diagnosis of both SAA [21-24] and CRP $[29,30]$. Compared with 0.67 (95\% CI 0.62$0.73)$ for the CRP test, the pooled sensitivity for the SAA test was better (0.78 (95\% CI 0.73-0.83)).

Pooled specificity for the SAA test was slightly lower than for the CRP test, which was 0.89 (95\% CI 0.84-0.92) versus 0.92 (95\% CI 0.89-0.95). Their difference was not statistically significant $(P<0.05)$. However, the pooled diagnostic OR for the SAA test was smaller than that for the CRP test: 54.95 (95\% CI 6.25-483.10) versus 77.16 (95\% CI 9.79-248.21). Although this difference was statistically significant $(P>$ $0.05)$, the $Q^{*}$ value for the SAA test was almost the same as that for the CRP test (0.89 VS 0.84), when the SROC curves for SAA and CRP tests were plotted, respectively. Meanwhile, the AUC for the detection of neonatal sepsis in the SAA 


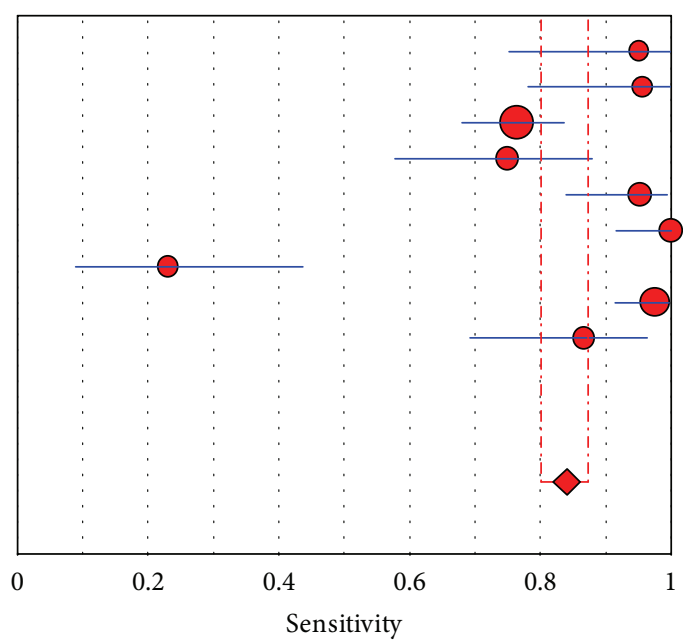

Enguix et al. 2001

Sensitivity $(95 \% \mathrm{CI})$

Arnon et al. 2007

$0.95(0.75-1.00)$

$0.96(0.78-1.00)$

Cetinkaya et al. $2009 \quad 0.76(0.68-0.84)$

Yildiz et al. 2008

$0.75(0.58-0.88)$

Arnon et al. 2005

$0.95(0.84-0.99)$

Arnon et al. 2002

$1.00(0.92-1.00)$

Edgar et al. 2010

$0.23(0.09-0.44)$

Mostafa et al. 2011

$0.98(0.91-1.00)$

Mohsen et al. 2012

$0.87(0.69-0.96)$

Pooled sensitivity $=0.84$ ( 0.80 to 0.87$)$

$\chi^{2}=95.82 ; \mathrm{df}=8(P=0.0000)$

Sensitivity

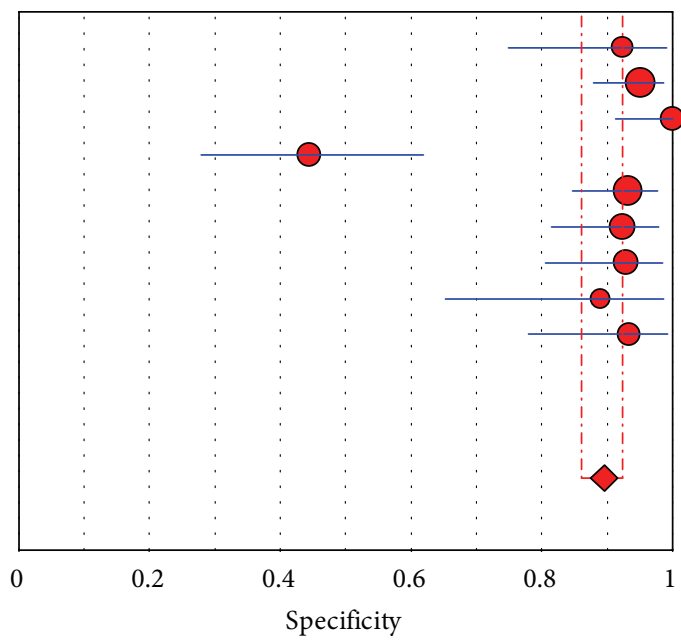

Enguix et al. 2001

Specificity $(95 \% \mathrm{CI})$

Arnon et al. 2007

$0.92(0.75-0.99)$

$0.95(0.88-0.99)$

$1.00(0.91-1.00)$

Çetinkaya et al. 2009

$0.44(0.28-0.62)$

$0.93(0.85-0.98)$

$0.92(0.81-0.98)$

$0.93(0.81-0.99)$

$0.89(0.65-0.99)$

$0.93(0.78-0.99)$

Pooled specificity $=0.89(0.86$ to 0.92$)$

$\chi^{2}=59.34 ; \mathrm{df}=8(P=0.0000)$

Inconsistency $\left(I^{2}\right)=86.5 \%$

FIgURE 3: Forest plot [46] for sensitivity and specificity of the SAA test to diagnose neonatal sepsis at the first suspicion of sepsis.

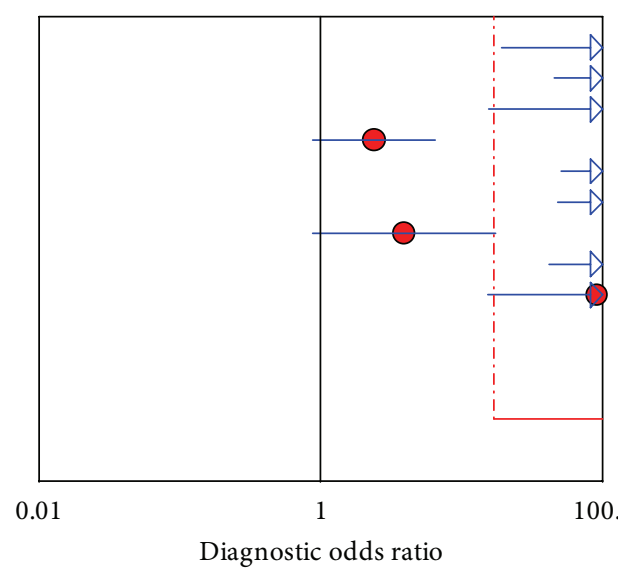

Enguix et al. 2001

Diagnostic OR $(95 \%$ CI)

Arnon et al. $2007 \quad 423.50(45.00-3,985.85)$

Çetinkaya et al. $2009 \quad 259.47(15.48-4,350.30)$

Yildiz et al. $2008 \quad 2.40(0.88-6.53)$

Arnon et al. $2005 \quad 272.00(50.41-1,467.77)$

Arnon et al. 2002

$916.11(47.92-17,514.22)$

Edgar et al. $2010 \quad 3.90(0.88-17.25)$

Mostafa et al. $2011 \quad 320.00(41.94-2,441.73)$

Mohsen et al. 2012

$91.00(15.36-539.26)$

Random effects model

Pooled diagnostic odds ratio $=91.84(16.78$ to 502.80$)$

Cochran- $Q=60.67 ; \mathrm{df}=8(P=0.0000)$

Inconsistency $\left(I^{2}\right)=86.8 \%$

$\tau^{2}=5.6399$

FIGURE 4: Forest plot for diagnostic OR of the SAA test to diagnose neonatal sepsis at the first suspicion of sepsis. 


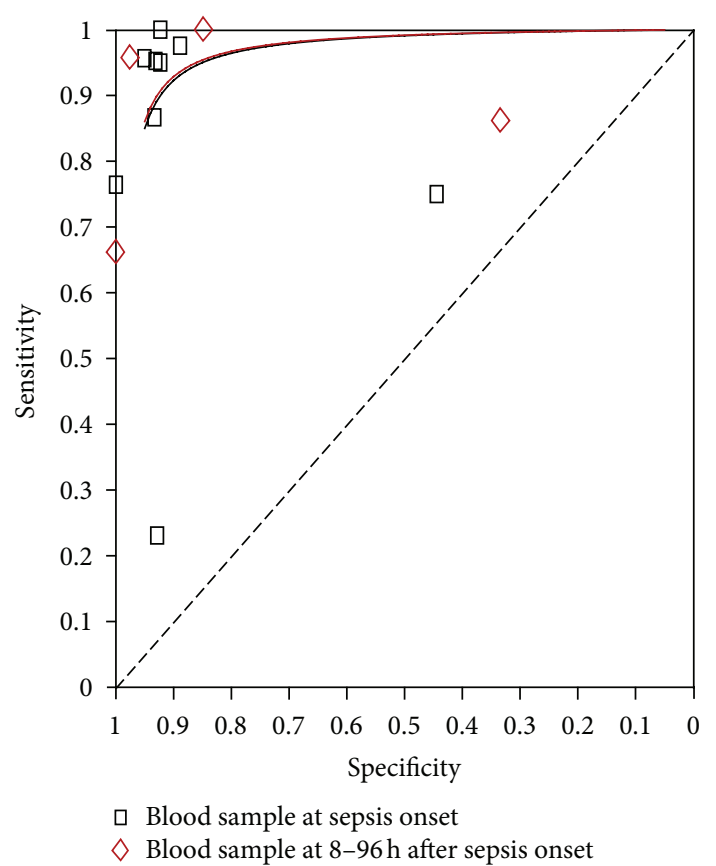

FIGURE 5: Summary receiver operating characteristic (SROC) curve of the SAA test for the diagnosis of neonatal sepsis.

test is larger than that in the CRP test (0.95 VS 0.91), which means that the SAA test is slightly better than CRP test for the diagnosis of NS by judging from the whole accuracy.

Two studies from included papers were selected to evaluate the use of the SAA and PCT for NS [23, 31]. The pooled sensitivity for the SAA test is slightly lower than for the PCT (0.78 (95\% CI 0.73-0.82) versus 0.89 (95\% CI 0.78-0.96)), but value of their specificity are similar to each other $(0.87$ versus 0.87 ). The pooled diagnosis OR for the SAA test was also almost similar to that for the PCT test (59.74 (95\% CI 6.08$586.65)$ versus 60.17 (95\% CI 8.09-447.31)). Since current trials are not sufficient to evaluate the use of SAA and PCT, the SROC curve for the PCT test cannot be plotted, and the $Q^{*}$ value and the AUC failed to be calculated.

3.4. Analysis of Heterogeneity. In a meta-analysis of diagnostic test, heterogeneity is an important issue to understand the possible factors that influence accuracy diagnosis and estimate the appropriateness of statistical pooling of results from various studies. Variations are brought by several factors, such as the cutoff, assay method, and the age of patients.

In fact, heterogeneity may not be entirely avoided in meta-analysis, so it is necessary for us to explore the reason and extent of heterogeneity. Generally, one of the most important sources of heterogeneity is the threshold effect in a diagnostic study. So, we firstly explored the threshold effect, which was evaluated with the Spearman correlation coefficient with Moses' model weighted by inverse variance. We found that there was no statistically significant difference (Spearman's correlation coefficient $=0.133, P$-value $=0.731>$ $0.5)$. The source of heterogeneity was explored by metaregression analysis. The results show that cutoff $(\geq 10 \mathrm{mg} / \mathrm{L})$ is the largest factor $(\mathrm{RDOR}$ (relative diagnostic odds ratio $)=$ $14.47, P$-value $=0.0963)$, while the effects of the age $(\mathrm{RDOR}=$ $0.35, P$-value $=0.591)$, assay method $(\mathrm{RDOR}=0.08, P$ value $=0.2405)$, region $(\mathrm{RDOR}=0.5, P$-value $=0.4997)$, and QUADAS $($ RDOR $=1.54, P$-value $=0.8076)$ are small. The sensitivity analysis was also performed to identify further analysis of these sources. Homogeneity $\left(I^{2}=0 \%\right)$ was showed among studies when we removed studies of Yildiz et al., 2008 [23], and Edgar, 2010 [24], including relatively small cutoff. If we only collect those studies in the diagnosis of lateonset neonates sepsis (postnatal age $>72 \mathrm{~h}$ ), heterogeneity $\left(I^{2}=89.9 \%\right)$ was found. When the study of Yildiz et al., 2008 [23] was removed, the remaining studies also showed homogeneity $\left(I^{2}=0 \%\right)$. As the amount of the included studies is too small, we cannot do further analysis of other factors, such as subgroup analysis. We except that our results will become more convincing if more and more studies about the SAA test of the diagnosis of neonatal sepsis are published in the future.

3.5. Publication Bias. The Deeks test [32] was performed to detect publication bias by using the Stata 11 software. As shown in Figure 8, this was not statistically significant for the studies of the SAA test in the diagnosis of neonatal sepsis $(P=0.406>0.05)$. So, the result indicates no potential for publication bias. However, our included studies are so few that this result may be biased.

\section{Discussion}

Neonatal sepsis is the most common cause of neonatal deaths with high mortality; thus, its identification is vital to improve bad results. Clinical signs are subtle and nonspecific, and laboratory tests including blood culture are not always reliable [33]. So far, many markers for NS have been suggested, such as CRP, PCT, IL-6, and TNF- $\alpha$. However, a single biomarker is not sufficiently reliable for identification of NS at present. More researchers focus on the combination of different biomarkers in different clinical settings and hope to achieve clearer conclusions $[34,35]$. Therefore, it is necessary for us to clearly study important markers for future research of NS. Here, we demonstrated that the SAA test shows appropriate accuracy for the diagnosis of NS.

An ideal biomarker should have high sensitivity and specificity, that is, have a high diagnostic accuracy [36]. In this meta-analysis, pooled sensitivity of the SAA test was 0.84 for the diagnosis of NS, the pooled specificity was 0.89 , and $Q^{*}$ value was 0.91 at the first suspicion of sepsis; then, 8$96 \mathrm{~h}$ after the first suspicion of sepsis, a pooled sensitivity of the SAA test was 0.78 for the diagnosis of NS, the pooled specificity was 0.84 , and $Q^{*}$ value was 0.91 . These results show that the SAA test is a valuable biomarker and also has a long diagnostic cycle for the diagnosis of NS.

Certainly, the limitations of our work should be considered. This meta-analysis only contained nine studies, though we tried our best to retrieve appropriate papers. In addition, we divided the sample into onset and 8-96 h after onset entirely based on characteristics of the sample, which accords with clinical facts, while the sample was divided into early-onset and late-onset neonatal sepsis generally. Different 


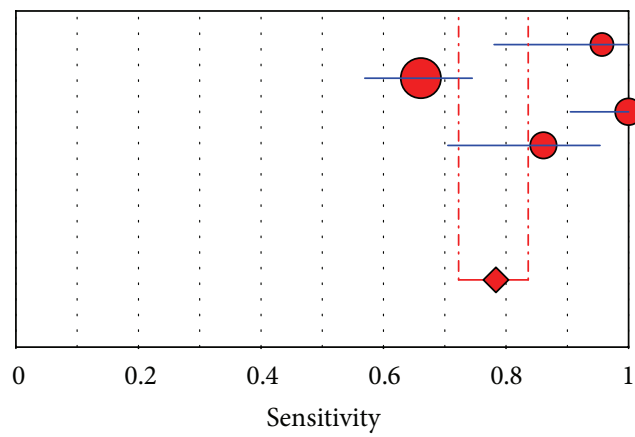

Arnon et al. 2007

Sensitivity (95\% CI)

Cetinkaya et al. $2009 \quad 0.66(0.57-0.74)$

Arnon et al. $2005 \quad 1.00(0.91-1.00)$

Yildiz et al. $2008 \quad 0.86(0.71-0.95)$

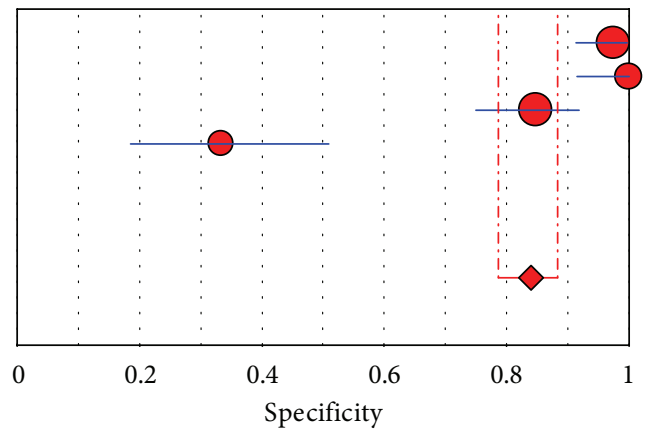

Arnon et al. 2007

Çetinkaya et al. 2009

Arnon et al. 2005

Yildiz et al. 2008

Specificity (95\% CI)

$0.98(0.91-1.00)$

$1.00(0.92-1.00)$

$0.85(0.75-0.92)$

$0.33(0.19-0.51)$

Pooled specificity $=0.84(0.79$ to 0.88$)$

$\chi^{2}=77.13 ; \mathrm{df}=3(P=0.0000)$

Inconsistency $\left(I^{2}\right)=96.1 \%$

FIGURE 6: Forest plots for sensitivity and specificity of the SAA test to diagnose neonatal sepsis at 8-96 h after the first suspicionof sepsis.

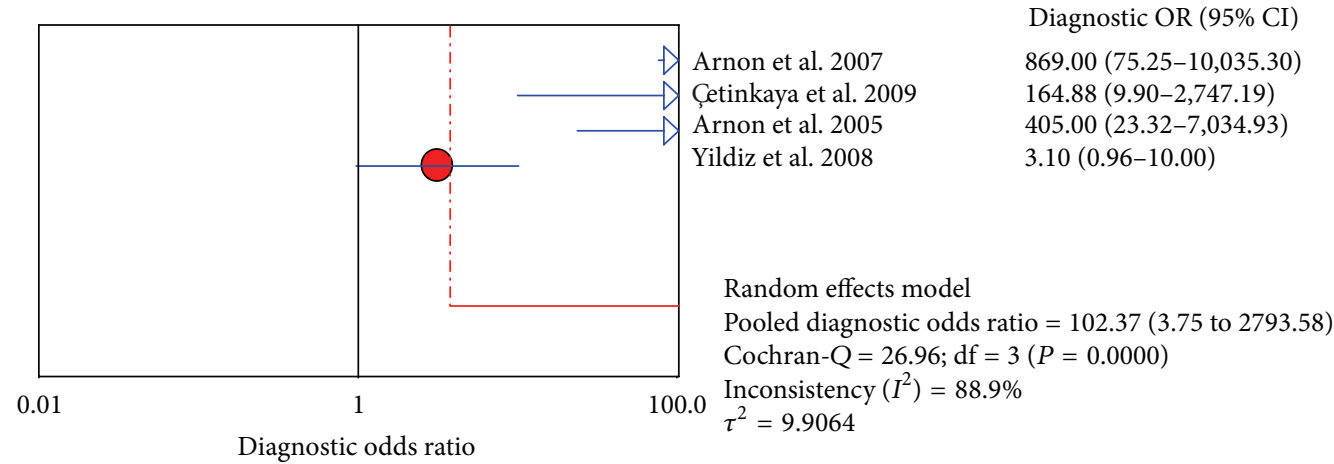

FIGURE 7: Forest plot for diagnostic OR of the SAA test to diagnose neonatal sepsis at 8-96 h after the first suspicion of sepsis.

cut-off values, ranging from 1 to $75.17 \mathrm{mg} / \mathrm{L}$, exist in the included studies. Publication bias might be generally difficult to be avoided in the meta-analysis, so we tried to include more studies and even adverse studies about the accuracy of the diagnosis of SAA to reduce the bias. To explore the reasons for heterogeneity, we considered the diagnostic cut-off point, which may partially explain this heterogeneity because of differences between studies. Therefore, we suspected that heterogeneity will be reduced by considering more studies for the SAA test, which might reduce the effect of different sampling, clinical settings, and other heterogeneity-caused factors.

CRP, a traditional useful marker, has been applied in clinic [37]. It is triggered by cytokines IL- 6 , TNF- $\alpha$, and so forth and is a late marker of neonatal sepsis which increases evidently at $24 \mathrm{~h}$ after sepsis onset $[22,38]$. Its sensitivity was
$30 \%-97 \%$, and its specificity ranged from $75 \%$ to $100 \%$ [39]. In included studies of the meta-analysis, the sensitivity of SAA varied from $26 \%$ to $100 \%$, and specificity varied from $44 \%$ to $100 \%$. To be truth, it is difficult to evaluate which marker is good or not. Lannergård et al. [40] also thought that there are positive correlations between SAA and CRP in infectious diseases. However, SAA rises earlier and more sharply than CRP, especially during the first $24 \mathrm{~h}$ after sepsis onset $[41,42]$, and it has showed a moderate accuracy at 8$96 \mathrm{~h}$ after the first suspicion of sepsis in our meta-analysis, which means that SAA is useful in the diagnosis of NS.

In addition, PCT is also a useful marker which shows better accuracy than CRP for the diagnotic of NS in some aspects [16]. In our meta-analysis, the pooled diagnotic OR for the SAA test was also almost similar to that for the PCT test (59.74 versus 60.17). So, we believe that SAA is 


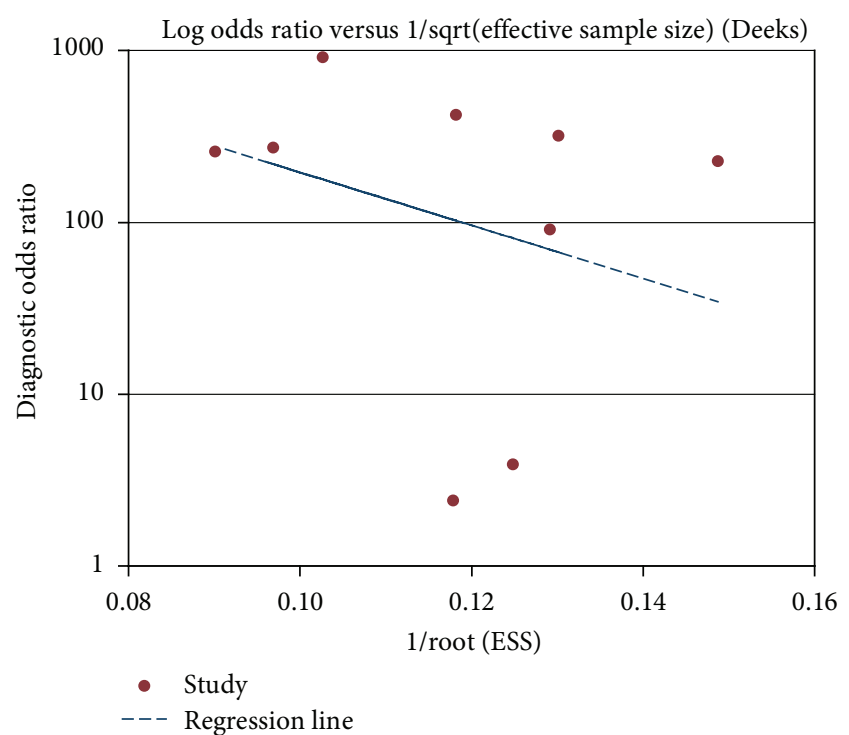

FIGURE 8: Test for the assessment of potential publication bias in the SAA test for the diagnosis of neonatal sepsis.

also a useful marker for the diagnosis of NS like CRP, PCT. Certainly, SAA cannot be replaced easily because it has a prognostic value as early as eight hours after the onset and before clinical signs. For example, des-arginine variants of SAA were identified as the most promising biomarkers, which can make neonatologists withhold treatment in $45 \%$ of nonsepsis neonates [43]. Moreover, SAA can be combined with sICAM-1, CRP, and sE-selectin to improve the diagnosis results, and it can be also added to PCT and CRP, which may increase the rate of sepsis diagnosis by about $10 \%[24,29]$. We believe that its usefulness will be showed if we evaluate it in combination with other markers or perform a clearer research on it alone in future studies.

In summary, SAA showed moderate accuracy and a longer diagnostic cycle in the diagnosis of neonatal sepsis. Furthermore, the SAA test has showed better accuracy than the CRP test for the diagnosis of neonatal sepsis in the first suspicion of sepsis. It not only has higher accuracy at the first suspicion of sepsis, but also keeps this usefulness at 8-96 after the first suspicion of sepsis. Accordingly, we believe that the combination of SAA with CRP and PCT will improve the diagnosis. To further analyze the diagnostic accuracy of the SAA test for the diagnosis of NS and correlation with other biomarkers in depth, follow-up clinical validation is needed.

\section{Authors' Contribution}

Haining Yuan and Jie Huang have equally contributed to this work.

\section{Acknowledgments}

This work was supported by the National Natural Science Foundation of China Grants (81272143, 21203131), the Natural Science Foundation of Jiangsu Province (K200509), the
Natural Science Foundation of the Jiangsu Higher Education Institutions of China (12KJB180014), Jiangsu Innovation Team Grant LJ201141, and Program of Innovative and Entrepreneurial Talent.

\section{References}

[1] S. A. Qazi and B. J. Stoll, "Neonatal sepsis: a major global public health challenge," Pediatric Infectious Disease Journal, vol. 28, no. 1, supplement, pp. S1-S2, 2009.

[2] Y.-J. Shin, M. Ki, and B. Foxman, "Epidemiology of neonatal sepsis in South Korea," Pediatrics International, vol. 51, no. 2, pp. 225-232, 2009.

[3] S. Vergnano, M. Sharland, P. Kazembe, C. Mwansambo, and P. T. Heath, "Neonatal sepsis: an international perspective," Archives of Disease in Childhood: Fetal and Neonatal Edition, vol. 90, no. 3, pp. F220-F224, 2005.

[4] E. Persson, B. Trollfors, L. L. Brandberg, and I. Tessin, "Septicaemia and meningitis in neonates and during early infancy in the Göteborg area of Sweden," Acta Paediatrica, vol. 91, no. 10, pp. 1087-1092, 2002.

[5] V. Sundaram, P. Kumar, S. Dutta et al., "Blood culture confirmed bacterial sepsis in neonates in a north Indian tertiary care center: changes over the last decade," Japanese Journal of Infectious Diseases, vol. 62, no. 1, pp. 46-50, 2009.

[6] P. C. Ng, "Clinical trials for evaluating diagnostic markers of infection in neonates," Biology of the Neonate, vol. 87, no. 2, pp. 111-112, 2005.

[7] M. W. Weber, J. B. Carlin, S. Gatchalian et al., "Predictors of neonatal sepsis in developing countries," The Pediatric Infectious Disease Journal, vol. 22, no. 8, pp. 711-717, 2003.

[8] N. Tripathi, C. M. Cotten, and P. B. Smith, "Antibiotic use and misuse in the neonatal intensive care unit," Clinics in Perinatology, vol. 39, no. 1, pp. 61-68, 2012.

[9] I. M. Stefanovic, "Neonatal sepsis," Biochemia Medica (Zagreb), vol. 21, no. 3, pp. 276-281, 2011.

[10] U. K. Mishra, S. E. Jacobs, L. W. Doyle, and S. M. Garland, "Newer approaches to the diagnosis of early onset neonatal sepsis," Archives of Disease in Childhood: Fetal and Neonatal Edition, vol. 91, no. 3, pp. F208-F212, 2006.

[11] P. C. Ng and H. S. Lam, "Diagnostic markers for neonatal sepsis," Current Opinion in Pediatrics, vol. 18, no. 2, pp. 125-131, 2006.

[12] C. Pierrakos and J.-L. Vincent, "Sepsis biomarkers: a review," Critical Care, vol. 14, no. 1, article R15, 2010.

[13] E. Bilavsky, H. Yarden-Bilavsky, S. Ashkenazi, and J. Amir, "C-reactive protein as a marker of serious bacterial infections in hospitalized febrile infants," Acta Paediatrica, International Journal of Paediatrics, vol. 98, no. 11, pp. 1776-1780, 2009.

[14] A. Kordek, M. Hałasa, and W. Podraza, "Early detection of an early onset infection in the neonate based on measurements of procalcitonin and C-reactive protein concentrations in cord blood," Clinical Chemistry and Laboratory Medicine, vol. 46, no. 8, pp. 1143-1148, 2008.

[15] J. P. S. Caldas, S. T. M. Marba, M. H. S. L. Blotta, R. Calil, S. S. Morais, and R. T. D. Oliveira, "Accuracy of white blood cell count, C-reactive protein, interleukin-6 and tumor necrosis factor alpha for diagnosing late neonatal sepsis," Jornal de Pediatria, vol. 84, no. 6, pp. 536-542, 2008.

[16] Z. Yu, J. Liu, Q. Sun, Y. Qiu, S. Han, and X. Guo, “The accuracy of the procalcitonin test for the diagnosis of neonatal sepsis: 
a meta-analysis," Scandinavian Journal of Infectious Diseases, vol. 42, no. 10, pp. 723-733, 2010.

[17] B. S. Naher, M. A. Mannan, K. Noor, and M. Shahiddullah, "Role of serum procalcitonin and C-reactive protein in the diagnosis of neonatal sepsis," Bangladesh Medical Research Council Bulletin, vol. 37, no. 2, pp. 40-46, 2011.

[18] S. Urieli-Shoval, R. P. Linke, and Y. Matzner, "Expression and function of serum amyloid A, a major acute-phase protein, in normal and disease states," Current Opinion in Hematology, vol. 7, no. 1, pp. 64-69, 2000.

[19] D. B. Jovanovic, "Clinical importance of determination of serum amyloid A," Srpski Arhiv Za Celokupno Lekarstvo, vol. 132, no. 78, pp. 267-271, 2004.

[20] S. Arnon, I. Litmanovitz, R. Regev, M. Lis, R. ShainkinKestenbaum, and T. Dolfin, "The prognostic virtue of inflammatory markers during late-onset sepsis in preterm infants," Journal of Perinatal Medicine, vol. 32, no. 2, pp. 176-180, 2004.

[21] S. Arnon, I. Litmanovitz, R. H. Regev, S. Bauer, R. ShainkinKestenbaum, and T. Dolfin, "Serum amyloid A: an early and accurate marker of neonatal early-onset sepsis," Journal of Perinatology, vol. 27, no. 5, pp. 297-302, 2007.

[22] S. Arnon, I. Litmanovitz, R. Regev et al., "Serum amyloid A protein is a useful inflammatory marker during late-onset sepsis in preterm infants," Biology of the Neonate, vol. 87, no. 2, pp. 105110, 2005.

[23] B. Yildiz, B. Ucar, M. A. Aksit et al., "Serum amyloid A, procalcitonin, tumor necrosis factor- $\alpha$, and interleukin- $1 \beta$ levels in neonatal late-onset sepsis," Mediators of Inflammation, vol. 2008, Article ID 737141, 7 pages, 2008.

[24] J. D. M. Edgar, V. Gabriel, J. R. Gallimore, S. A. McMillan, and J. Grant, "A prospective study of the sensitivity, specificity and diagnostic performance of soluble intercellular adhesion molecule 1, highly sensitive C-reactive protein, soluble E-selectin and serum amyloid A in the diagnosis of neonatal infection," $B M C$ Pediatrics, vol. 10, article 22, 2010.

[25] P. Whiting, A. W. S. Rutjes, J. B. Reitsma, P. M. M. Bossuyt, and J. Kleijnen, "The development of QUADAS: a tool for the quality assessment of studies of diagnostic accuracy included in systematic reviews," BMC Medical Research Methodology, vol. 3, article 1, 2003.

[26] A. S. Glas, J. G. Lijmer, M. H. Prins, G. J. Bonsel, and P. M. M. Bossuyt, "The diagnostic odds ratio: a single indicator of test performance," Journal of Clinical Epidemiology, vol. 56, no. 11, pp. 1129-1135, 2003.

[27] J. P. T. Higgins, S. G. Thompson, J. J. Deeks, and D. G. Altman, "Measuring inconsistency in meta-analyses," British Medical Journal, vol. 327, no. 7414, pp. 557-560, 2003.

[28] S. D. Walter, "Properties of the summary receiver operating characteristic (SROC) curve for diagnostic test data," Statistics in Medicine, vol. 21, no. 9, pp. 1237-1256, 2002.

[29] M. Çetinkaya, H. Özkan, N. Köksal, S. Çelebi, and M. Hacimustafaoğlu, "Comparison of serum amyloid A concentrations with those of $\mathrm{C}$-reactive protein and procalcitonin in diagnosis and follow-up of neonatal sepsis in premature infants," Journal of Perinatology, vol. 29, no. 3, pp. 225-231, 2009.

[30] L. M. Mohsen et al., "Study on diagnostic value of serum amyloid A protein during late- onset sepsis in preterm and full term neonates," Australian Journal of Basic and Applied Sciences, vol. 6, no. 12, pp. 530-536, 2012.

[31] A. Enguix, C. Rey, A. Concha, A. Medina, D. Coto, and M. A. Diéguez, "Comparison of procalcitonin with C-reactive protein and serum amyloid for the early diagnosis of bacterial sepsis in critically ill neonates and children," Intensive Care Medicine, vol. 27, no. 1, pp. 211-215, 2001.

[32] J. J. Deeks, P. Macaskill, and L. Irwig, "The performance of tests of publication bias and other sample size effects in systematic reviews of diagnostic test accuracy was assessed," Journal of Clinical Epidemiology, vol. 58, no. 9, pp. 882-893, 2005.

[33] J. S. Gerdes, "Diagnosis and management of bacterial infections in the neonate," Pediatric Clinics of North America, vol. 51, no. 4, pp. 939-959, 2004.

[34] S. Arnon and I. Litmanovitz, "Diagnostic tests in neonatal sepsis," Current Opinion in Infectious Diseases, vol. 21, no. 3, pp. 223-227, 2008.

[35] Y. Fan and J. L. Yu, "Umbilical blood biomarkers for predicting early-onset neonatal sepsis," World Journal of Pediatrics, vol. 8, no. 2, pp. 101-108, 2012.

[36] J. C. Marshall and K. Reinhart, "Biomarkers of sepsis," Critical Care Medicine, vol. 37, no. 7, pp. 2290-2298, 2009.

[37] M. M. Levy, M. P. Fink, J. C. Marshall et al., "2001 SCCM/ ESICM/ACCP/ATS/SIS International Sepsis Definitions Conference," Intensive Care Medicine, vol. 29, no. 4, pp. 530-538, 2003.

[38] B. Janković, D. Veljković, S. Pasić, Z. Rakonjac, D. Jevtić, and J. Martić, "C-reactive protein and cytokines in the diagnosis of neonatal sepsis," Medicinski pregled, vol. 59, no. 11-12, pp. 545549, 2006.

[39] T. Chan and F. Gu, "Early diagnosis of sepsis using serum biomarkers," Expert Review of Molecular Diagnostics, vol. 11, no. 5, pp. 487-496, 2011.

[40] A. Lannergård, A. Larsson, P. Kragsbjerg, and G. Friman, "Correlations between serum amyloid A protein and C-reactive protein in infectious diseases," Scandinavian Journal of Clinical and Laboratory Investigation, vol. 63, no. 4, pp. 267-272, 2003.

[41] C. Pizzini, M. Mussap, M. Plebani, and V. Fanos, "C-reactive protein and serum amyloid A protein in neonatal infections," Scandinavian Journal of Infectious Diseases, vol. 32, no. 3, pp. 229-235, 2000.

[42] M. Mussap, A. Noto, F. Cibecchini, and V. Fanos, "The importance of biomarkers in neonatology," Seminars in Fetal \& Neonatal Medicine, vol. 18, no. 1, pp. 56-64, 2013.

[43] P. C. Ng, I. L. Ang, R. W. K. Chiu et al., "Host-response biomarkers for diagnosis of late-onset septicemia and necrotizing enterocolitis in preterm infants," Journal of Clinical Investigation, vol. 120, no. 8, pp. 2989-3000, 2010.

[44] S. Arnon, I. Litmanovitz, R. Regev, M. Lis, R. ShainkinKestenbaum, and T. Dolfin, "Serum amyloid A protein in the early detection of late-onset bacterial sepsis in preterm infants," Journal of Perinatal Medicine, vol. 30, no. 4, pp. 329-332, 2002.

[45] M. S. Mostafa et al., "Serum amyloid A an early diagnostic marker for neonatal sepsis," Life Science Journal, vol. 8, no. 3, pp. 271-277, 2011.

[46] S. Lewis and M. Clarke, "Forest plots: trying to see the wood and the trees," British Medical Journal, vol. 322, no. 7300, pp. 14791480, 2001. 

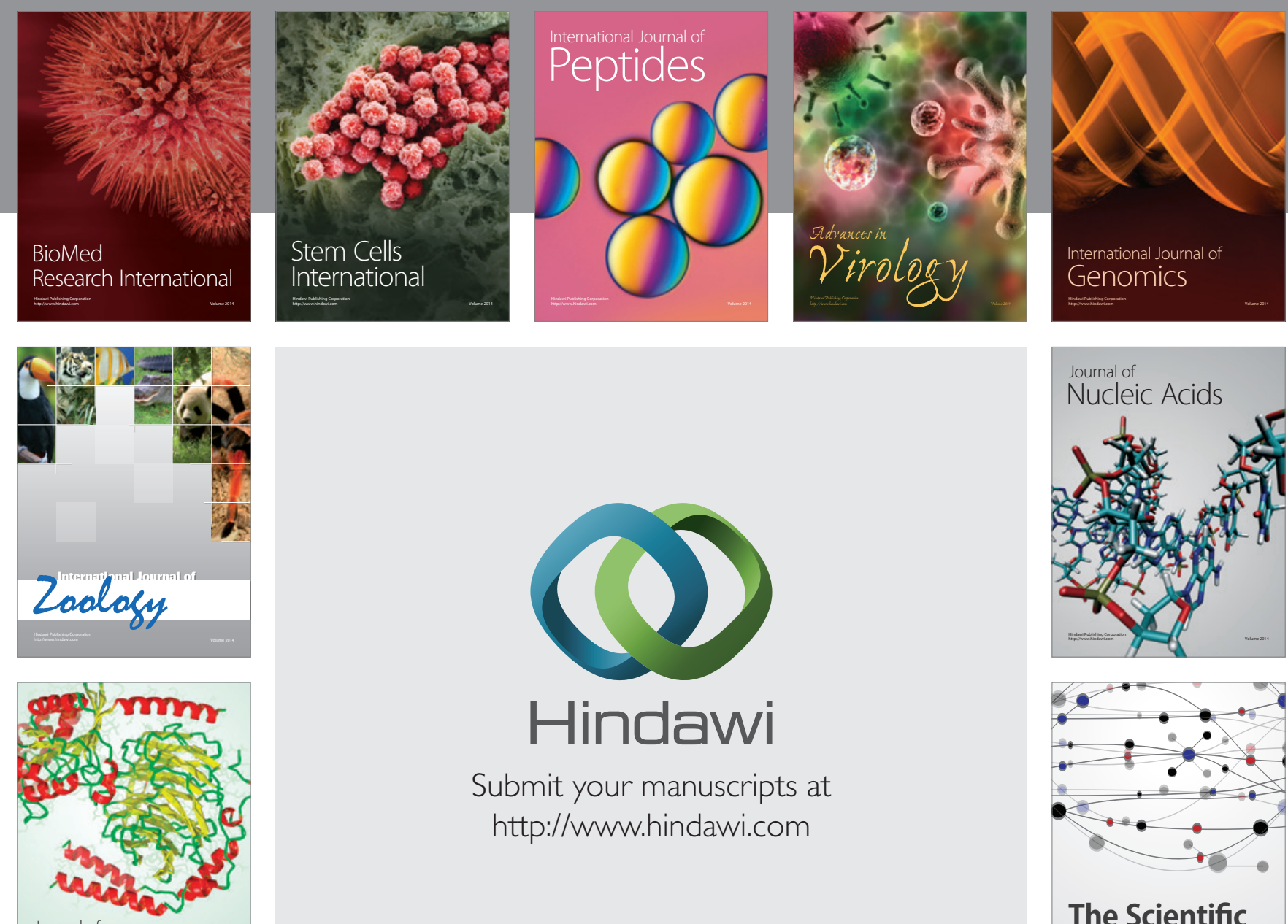

Submit your manuscripts at

http://www.hindawi.com

Journal of
Signal Transduction
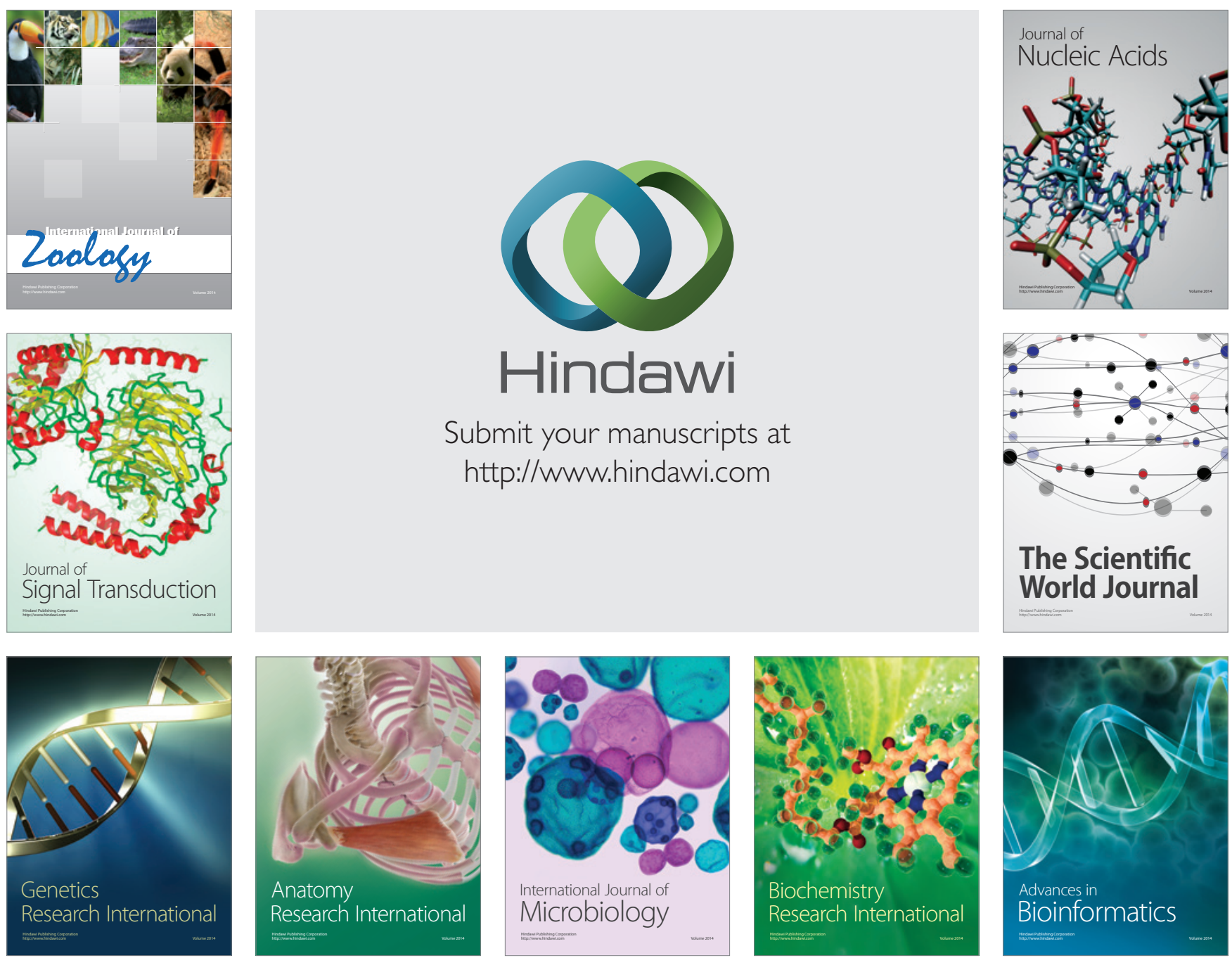

The Scientific World Journal
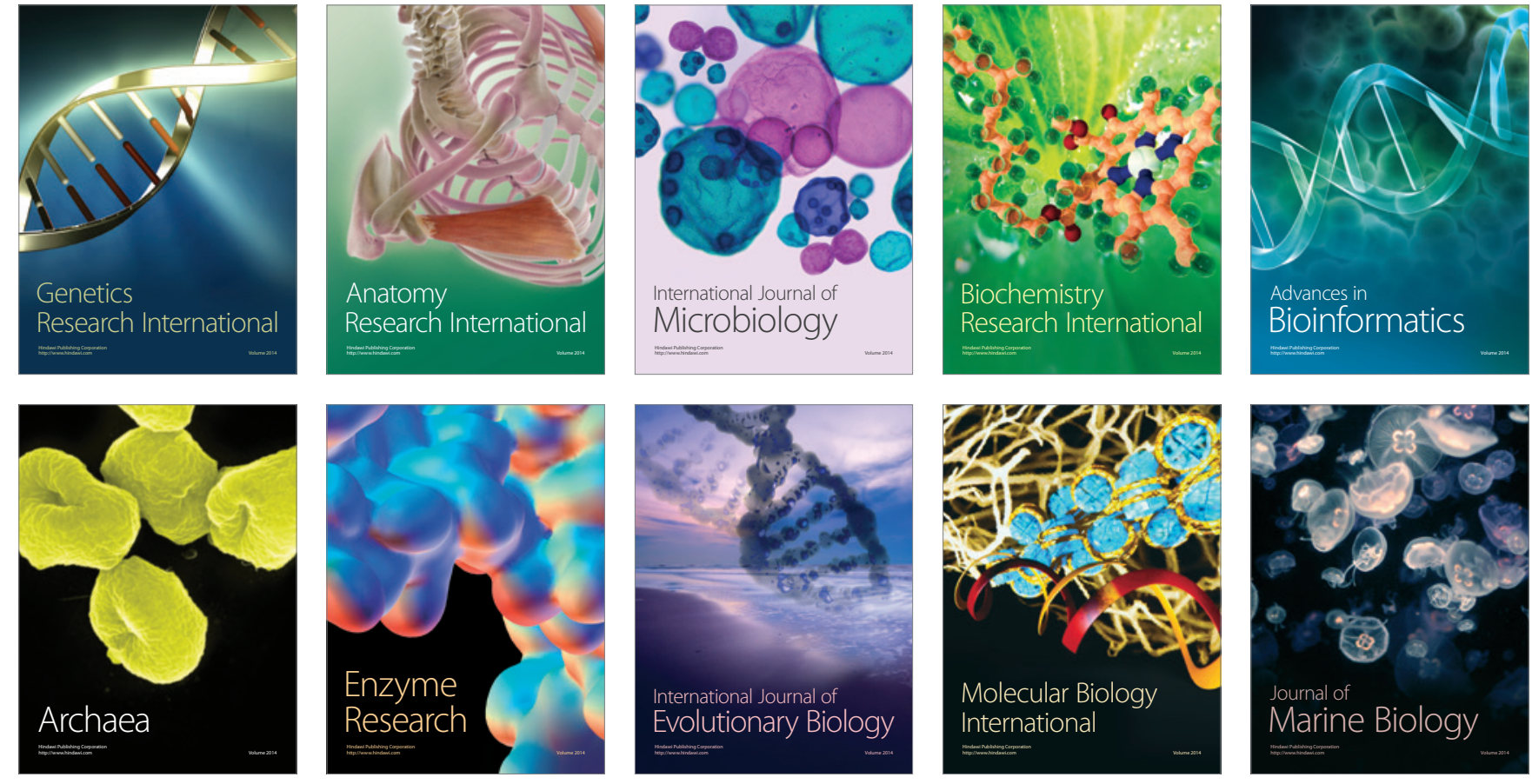